\title{
Patients with Mild Cognitive Impairment Show Lower Visual Short-Term Memory Performance in Feature Binding Tasks
}

\author{
Raju P. Sapkota ${ }^{a} \quad$ Ian van der Linde ${ }^{a, b} \quad$ Nirmal Lamichhane $^{c}$ \\ Tirthalal Upadhyayad Shahina Pardhan ${ }^{\mathrm{a}}$ \\ ${ }^{a}$ Vision and Eye Research Unit (VERU), Postgraduate Medical Institute, Faculty of Medical \\ Science, Anglia Ruskin University, Cambridge, UK; b Department of Computing and \\ Technology, Anglia Ruskin University, Cambridge, UK; ' ${ }^{C}$ Department of Neuropsychiatry, \\ Gandaki Medical College Teaching Hospital and BG Hospital and Research Center Pvt. Ltd, \\ Pokhara, Nepal; d Department of Medicine, Gandaki Medical College, Teaching Hospital, \\ Pokhara, Nepal
}

\section{Keywords}

Visual short-term memory $\cdot$ Mild cognitive impairment $\cdot$ Binding $\cdot$ Dementia

\begin{abstract}
Background: Early cognitive changes in people at risk of developing dementia may be detected using behavioral tests that examine the performance of typically affected brain areas, such as the hippocampi. An important cognitive function supported by the hippocampi is memory binding, in which object features are associated to create a unified percept. Aim: To compare visual short-term memory (VSTM) binding performance for object names, locations, and identities between a participant group known to be at higher risk of developing dementia (mild cognitive impairment $[\mathrm{MCI}]$ ) and healthily aging controls. Methods: Ten MCI and 10 control participants completed five VSTM tests that differed in their requirement of remembering bound or unbound object names, locations, and identities, along with a standard neuropsychological test (Addenbrooke's Cognitive Examination [ACE]-III). Results: The performance of the MCI participants was selectively and significantly lower than that of the healthily aging controls for memory tasks that required object-location or name-location binding. Conclusion: Tasks that measure unimodal (object-location) and crossmodal (namelocation) binding performance appear to be particularly effective for the detection of early cognitive changes in those at higher risk of developing dementia due to Alzheimer's disease.
\end{abstract}


Sapkota et al.: Patients with Mild Cognitive Impairment Show Lower Visual Short-Term Memory Performance in Feature Binding Tasks

\section{Introduction}

The human visual system is organized such that different features belonging to the same object, such as its color, shape, name, and location, are processed in different areas of the brain $[1,2]$. It is known, for example, that color, shape, and name features are processed via the ventral route, the neural pathway that projects forward from the primary visual cortex towards the temporal lobe (the so-called what pathway). Location features are processed via the dorsal route, the neural pathway that projects forward from the primary visual cortex towards the parietal lobe (the so-called where pathway) [2]. Some studies have augmented the where function of the dorsal route with how, based on the argument that this pathway is also involved in performing visually guided hand movements, such as reaching and grasping $[3,4]$. Communication between the dorsal and the ventral routes is supported by numerous neural interconnections [1,5-7].

Mechanisms in the brain exist to integrate (bind) an object's visual and location features together to allow the perception of a unified entity [8]. These mechanisms are crucial in enabling us to remember the location and visual properties of objects in our physical environment, and they guide our behavior during everyday tasks such as reading a road map, looking for a misplaced item, or remembering where we have parked our car, by avoiding unnecessary repeated viewing of the same locations.

Our ability to bind object features together during a visual short-term memory (VSTM) task, which makes use of a temporary memory store for visual information [9], is thought to be supported by the hippocampus and surrounding brain structures such as the entorhinal and perirhinal cortices $[10,11]$. These structures are known to deteriorate with age $[12,13]$, and are believed to be affected early in the progression of Alzheimer's disease (AD) [14], a degenerative brain condition that affects memory and other cognitive functions, leading to dementia.

Mild cognitive impairment (MCI) is a condition that represents the transitional (preclinical) stage that distinguishes the deterioration of cognitive function associated with normal aging from dementia [3]. Individuals with $\mathrm{MCI}$ often complain of memory problems (amnestic) noticeable to themselves and their close relatives, but these changes are not significant enough to adversely affect their day-to-day functioning, and they do not fulfill the clinical criteria for dementia [15]. Test scores of MCI participants are typically found to be in the range of 75-88/100 in Addenbrooke's Cognitive Examination (ACE) [16, 17] and 18-24/30 in the Mini-Mental State Examination (MMSE) [18]. ACE-III is a 100-item questionnaire that measures overall cognitive performance (attention, memory, language, fluency, and visuospatial skills). The MMSE uses a short (30-item) questionnaire, and is one of the most commonly used tests to screen for cognitive impairment. More than half of the individuals with MCI progress to developing dementia within 5 years [19]. As patients with MCI are at a higher risk of developing dementia of AD type, and since VSTM binding is believed to recruit the same brain areas as are affected in early AD, we hypothesize that people with $\mathrm{MCI}$ will show characteristic changes in VSTM binding (object-location) and crossmodal binding (auditorily presented object name-location). Indeed, in several previous studies visuospatial memory has been advocated as a possible early marker for AD [20-23], although the experimental procedures presented to date are insufficiently developed (i.e., do not examine different forms of memory binding for isolating the specific functions that are most adversely affected) and lack appropriate clinical verification.

Paper-based tests for assessing dementia, such as ACE-III [16, 17], the MMSE [18], the Wechsler Memory Scale (WMS)-III [24], the Alzheimer's Disease Assessment Scale (ADAS) [25], and the Clinical Dementia Rating (CDR) [26] remain clinically dominant over procedures such as blood and imaging tests, primarily because they provide a direct measurement of an indi- 
vidual's cognitive capabilities. Furthermore, paper-based tests are of low cost, are non-invasive, and are relatively easy to administer, often comprising simple questionnaires or interviews that focus upon a patient's day-to-day activities and experiences. These tests are designed to tap into the activities of a wide range of cognitive functions, such as memory, reasoning, attention, and orientation, and hence provide an assessment of an individual's overall cognitive performance. Test scores become noticeably lower as the brain areas that support these cognitive abilities are ultimately affected (e.g., prefrontal cortex/frontal lobe). Whilst existing tests are suitable for assessing a wide range of cognitive abilities, these tests focus upon only a basic subset of VSTM functions, such as storage capacity and recency effects. In other words, these tests do not provide a more comprehensive assessment of higher-order VSTM abilities such as memory binding, which are likely to be affected earlier in people who may develop dementia prospectively (discussed above). The WMS-III and the Cambridge Neuropsychological Test Automated Battery-Paired Associative Learning $[27,28]$ do, to some degree, measure memory binding, but at a very basic object-location level. Hence, the majority of the routine cognitive tests used for assessing dementia may be better suited to establishing the severity of the condition following clinical onset, rather than enabling early (preclinical) detection [29].

In this study, we examined whether the performance profile produced in a battery of VSTM tasks, including memory binding, differs between $\mathrm{MCI}$ and normally aging control participants in order to assess the diagnostic utility of these tests in detecting the early cognitive changes linked to dementia of AD type.

\section{Subjects and Methods}

\section{Participants}

Ten older adults with MCI (mean age $=67.3$ years, $\mathrm{SD}=4.0$; mean educational level $=$ 11.10 years, $\mathrm{SD}=1.60$ ) and 10 normally aging older control adults (mean age $=63.9$ years, $\mathrm{SD}=2.73$; mean educational level $=11.60$ years, $\mathrm{SD}=1.07)$ participated. The participant groups did not differ significantly in age $(p=0.17)$ or educational level $(p=0.42)$. The participants gave their informed consent prior to taking part in the study.

The study protocol was approved by the Ethics Committee on Human Research at Gandaki Medical College, Pokhara, Nepal (approval No. 302845752). The participants were treated in accordance with applicable ethical guidelines that followed the tenets of the Declaration of Helsinki. The MCI participants were identified by a clinical neuropsychiatrist and a geriatric physician using the following inclusion/exclusion criteria.

Included were individuals $\geq 60$ years of age with an overall ACE-III score between 75 and 88 and a memory domain score of $<24$, i.e., amnestic MCI [16]. These individuals had normal or corrected-to-normal vision and no hearing impairment (self-reported), as well as the capacity to give informed consent for taking part in the study.

Excluded were

1. individuals with clinically established dementia (including dementia of AD type, dementia with Lewy bodies, frontotemporal dementia, and vascular dementia);

2. individuals with a history of stroke, traumatic brain injury, or degenerative diseases such as Parkinson and Huntington disease;

3. individuals with a history of profound depression, anxiety, and learning difficulties;

4. individuals with a history of chronic sleep problems, chronic alcohol or drug abuse, and related health problems; individuals with a history of epilepsy, brain tumor, or brain surgery.

Normally aging control participants ( $\geq 60$ years of age) were recruited from the community, and they had an overall ACE-III score $>88$. They also had normal or corrected-to- 
Sapkota et al.: Patients with Mild Cognitive Impairment Show Lower Visual Short-Term Memory Performance in Feature Binding Tasks

Fig. 1. Examples of stimuli.
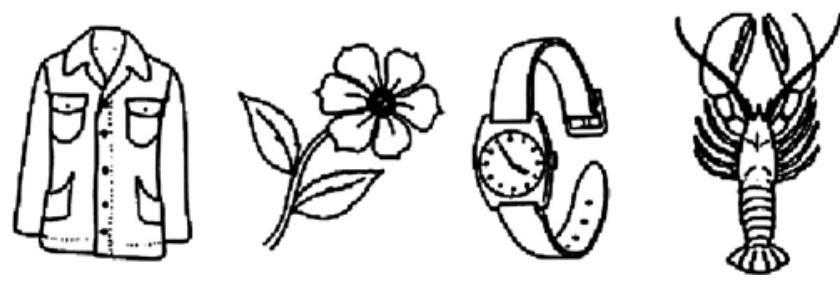

normal vision and no hearing impairment (self-reported) and possessed the capacity to give consent for taking part in the study.

All participants were non-native English speakers. All eligible participants were tested on the VSTM tasks by author R.P.S.

\section{Stimuli}

The stimuli comprised 180 line drawings of real-world objects [30], each subtending $2.5^{\circ}$ of the visual angle at $57 \mathrm{~cm}$. The stimuli belonged to one of 14 semantic categories (4-footed animals, birds, kitchen utensils, etc.). Example stimuli are shown in Figure 1. Nameable stimuli like these were used (rather than non-nameable novel objects) for ecological validity, and because, in some trials, our experimental procedure required cueing object locations by auditorily presented names (see Procedure). Stimulus presentation was controlled by MATLAB (Mathworks, Natick, MA, USA) with PsychToolbox/Video Toolbox extensions [31, 32]. The stimulus background was set to mid-gray.

\section{Apparatus}

The stimuli were displayed using a Sony laptop computer (model: PCG-71313M; Sony Corporation, Japan) with the screen resolution set at 1,366 $\times 768$ pixels and a refresh rate of $60 \mathrm{~Hz}$. The viewing distance was set to be approximately $57 \mathrm{~cm}$. The ambient light was held constant across trials and between participants. A computer mouse was used to enter the participants' responses.

\section{Procedure}

ACE-III was repeated for each participant before taking part in the VSTM experiments, to make sure that the participant still fulfilled the inclusion criteria at the time of testing (summarized below). The experimental procedures were preceded by a stimulus learning routine, during which all 180 stimuli were displayed sequentially in random order; the participants were asked to name each stimulus in English as it appeared. When the participants could not name/recognize a stimulus, the experimenter familiarized them with it by giving them a verbal prompt (its name). Next, stimuli that the participants could not originally name were presented again one at a time, and the participants were asked again to name them in English as they appeared. All participants were able to name all stimuli correctly in English before taking part in the VSTM tests.

\section{Test 1}

Test 1 (Fig. 2, test 1) measured the participants' memory for objects only. The following procedures were used: each trial began with a fixation cross displayed at the screen's center for $800 \mathrm{~ms}$. This ensured that all participants fixated upon a common screen position prior to the memory display. Next, either 2 or 4 line drawings of real-world objects (see Stimuli) were 


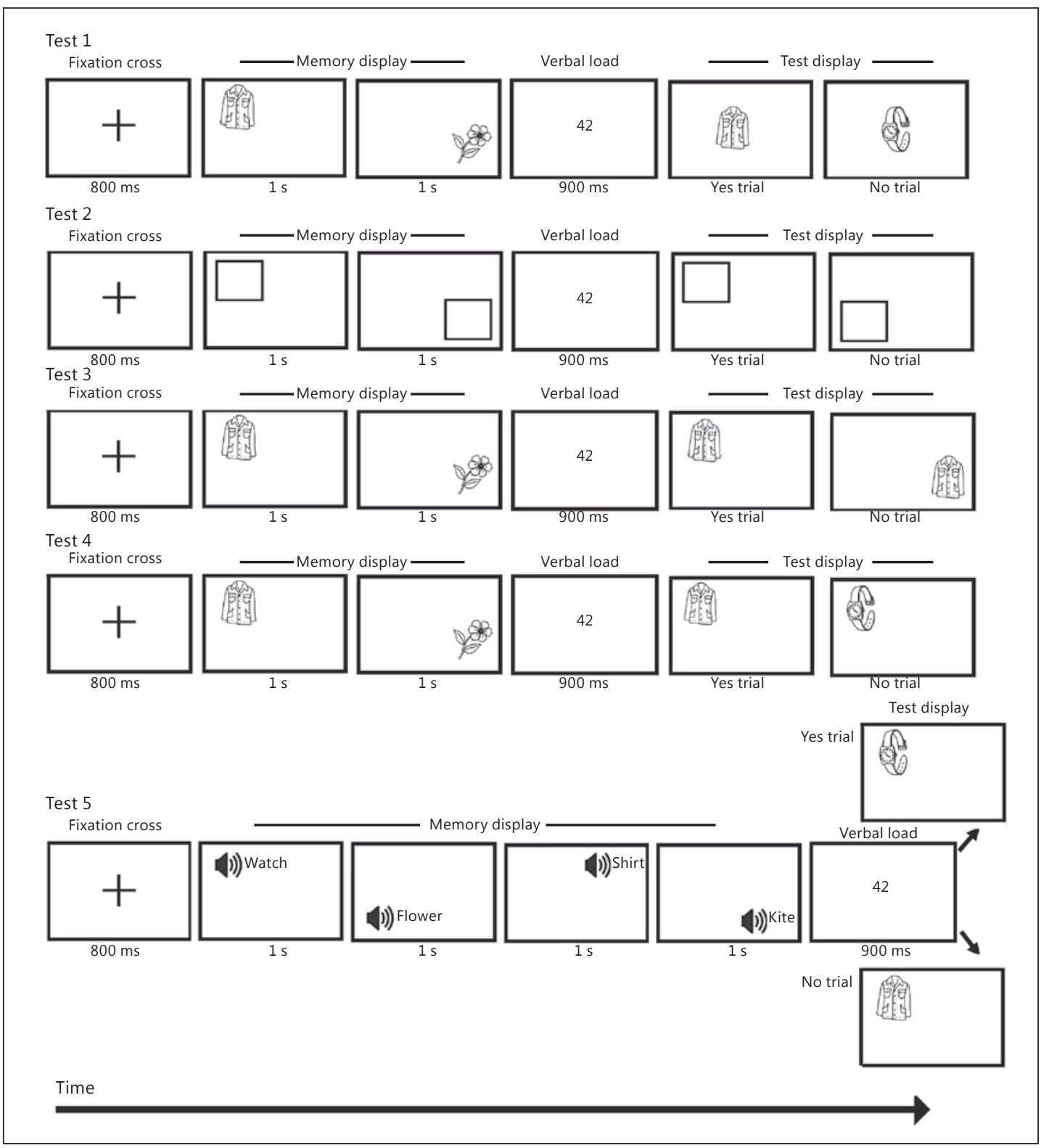

Fig. 2. Self-explanatory schematic diagram (not to scale) of the trial sequence used. Each rectangle represents the appearance of the computer screen at a given time. For tests $1-4$, only memory load 2 is represented. For test 5, memory load 4 is represented.

shown on the computer screen sequentially (i.e., one stimulus at a time), each for $1 \mathrm{~s}$, at random locations, which participants were asked to remember. We refer to this as the "memory display." This was followed by the presentation of a 2-digit number at the center of the display screen for $900 \mathrm{~ms}$. The participants were asked to read this number aloud to discourage verbal encoding of the visual stimuli $[33,34]$. Following this, a test display was presented in which either a previously shown object (yes trial) or a new object (no trial) was displayed at the center of the screen. The participants were required to identify whether the test object had been shown in the preceding memory display, and to give a yes/no verbal response. The responses were entered by the experimenter using the left and right buttons 
Sapkota et al.: Patients with Mild Cognitive Impairment Show Lower Visual Short-Term Memory Performance in Feature Binding Tasks

of a computer mouse for yes and no responses, respectively. The next trial commenced immediately after a response had been entered.

There were 16 trials in total. These were divided equally between the two memory loads (i.e., sequence length 2 and sequence length 4 ). For each memory load, there were an equal number of yes and no trials. In yes trials, the temporal positions used to present stimuli in the memory display were probed equally often (i.e., for sequence length 2 , each of the 2 temporal positions were tested twice; for sequence length 4 , each of the 4 temporal positions were tested once). The participants were instructed to concentrate on response accuracy rather than response speed.

Each participant completed a practice block of 6 trials before the experimental data were collected. The participants were permitted to rest whenever they wished by informing the experimenter, in which case the response they provided was not entered by the experimenter until they said they were ready for the next trial.

Test 2

Test 2 measured the participants' memory for location only (Fig. 2, test 2). The procedures were similar to those of test 1 , except that in the memory display, an empty square box $\left(2.5 \times 2.5^{\circ}\right)$ was shown sequentially at 2 or 4 random spatial positions on the computer screen. In the test display, a spatial marker $\left(2.5 \times 2.5^{\circ}\right.$ empty square box $)$ was shown either at one of the square locations cued in the memory display (yes trial), or at a new location not cued in the memory display (no trial). The participants were required to identify whether the location of the square box shown in the test display had been cued in the preceding memory display, and give a yes/no verbal response. The number of trials across memory loads (i.e., sequence length 2 and sequence length 4 ) and temporal positions was distributed similarly to test 1.

Test 3

Test 3 measured the participants' memory for object identity and location combined (i.e., object-location binding) (Fig. 2, test 3). The experimental procedures and the number and distribution of trials across memory loads were similar to test 1 , except that in yes trials, the test object (selected randomly from the memory display) was shown at its original location. In no trials, the test object was shown at a location occupied previously by a different memory object. Hence, memory for the binding of the target object to its location was required to perform the task successfully.

The participants responded yes/no verbally to indicate whether they believed the location of the test object was the same or different to the location at which it had been shown in the preceding memory display.

\section{Test 4}

Test 4 measured the participants' object memory with location priming (Fig. 2, test 4). Stimulus locations were repeated from memory to test display, but unlike in test 3 , in no trials a new object not previously used in the memory display was shown in the test display. This enabled us to determine whether any responses in test 3 were driven by priming effects due to stimulus locations being repeated. Trials across memory loads and temporal positions were distributed similarly to test 3 .

\section{Test 5}

Test 5 measured the participants' ability to bind an object's verbally spoken name to its location (crossmodal binding for name-location) (Fig. 2, test 5). The procedures were similar to those used in test 3 , except that instead of showing object drawings in the memory display, empty square boxes were shown one after another at 4 random locations (i.e., only sequence 
length 4 was used). While examining the empty boxes, the participants also heard object names spoken (loud enough) by the computer in English; the presentation of the auditory stimuli was synchronized with the presentation of the empty square boxes in the memory display. The test display comprised a line drawing of one of the objects which was named in the memory display; in yes trials, the test object was shown at the location at which its name was spoken when the square box had appeared at this location in the memory display. In no trials, the test object was shown at the location at which its name was spoken when the square box had appeared at a different location in the memory display. Hence, memory for binding of an object's name, presented auditorily, to its location was examined.

The participants responded yes/no verbally to indicate whether the image of the test display object was shown at the cued location at which its name was spoken in the memory display.

Each participant completed 6 practice trials, followed by 16 experimental trials. There were an equal number of yes and no trials. In yes trials, the temporal positions used to cue locations in the memory display were probed equally often (i.e., each of the 4 temporal positions were tested twice).

The test order was randomized for each participant. It took approximately $25 \mathrm{~min}$ for each participant to complete all tests, including completing ACE-III.

\section{Statistical Analysis}

The data were categorized according to signal detection theory measures [35]. Performance was analyzed in terms of percent correct responses. Hit rates across temporal positions were used to interpret recency effects. The $z$-scores of hit rate and false alarm rate were used to calculate response biases $(\beta)$. Between-group participant data were analyzed using 2 (participant groups) $\times 2$ (memory load) mixed ANOVAs or independent-samples $t$ tests, as required. Within-group participant data across temporal positions were analyzed using one-way repeated-measures ANOVA (with temporal position as a within-subject factor) or paired $t$ tests, as required. Where the assumption of sphericity was violated (identified using Mauchly's test), degrees of freedom were adjusted using the Greenhouse-Geisser procedure.

\section{Results}

The average ACE-III scores were found to be 79.30 (SD = 3.23) and $90.10(\mathrm{SD}=0.74)$ for the MCI and age-matched control participants, respectively; the difference was statistically significant $(t(10)=10.30, p<0.001)$. Similarly, a statistically significant difference was found between the participant groups for total ACE-III scores for the memory domain only $(t(18)=$ $2.67, p=0.02$ ), but not for other cognitive domains such as attention, language, fluency, and visuospatial skills $(p \geq 0.33)$.

The mean performances (percent correct responses) for tests 1-5 are shown in Table 1. Figure 3 shows the overall percent correct responses (averaged across the sequence lengths) for tests $1-5$. For object-only memory (test 1 ), a 2 (participant groups) $\times 2$ (memory loads) mixed ANOVA showed no difference in overall performance between the participant groups (Table 1). Although a significant effect of memory load (2 or 4 objects) on task performance was found for both participant groups (Table 2; MCI participants, $p=0.003$; controls, $p=$ $0.005)$, a significant difference in performance between the participant groups was not found for either memory loads (Table 1). The recency effects were not significant for either participant group with either memory load (Table 2).

For location-only memory (test 2), no difference in overall performance between the participant groups was found when averaged across the memory loads (Table 1). Similarly to 
Demementia
and Geriatric
Cognitive Disorders

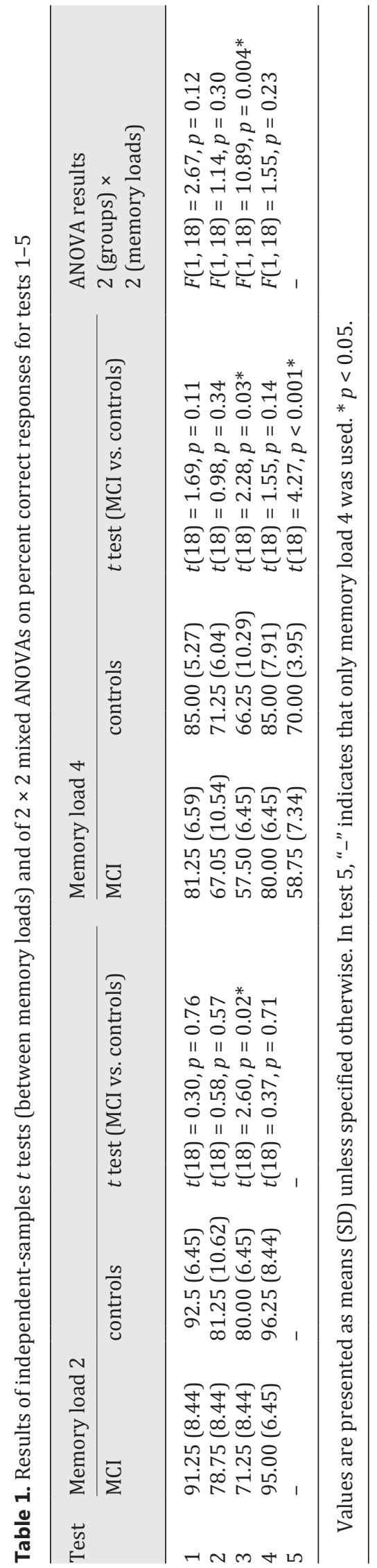

Sapkota et al.: Patients with Mild Cognitive Impairment Show Lower Visual Short-Term Memory Performance in Feature Binding Tasks 
Fig. 3. Overall percent correct responses of the mild cognitive impairment (MCI) and control participants in tests $1-5$. Error bars represent $\pm 1.96 \mathrm{SE}$.

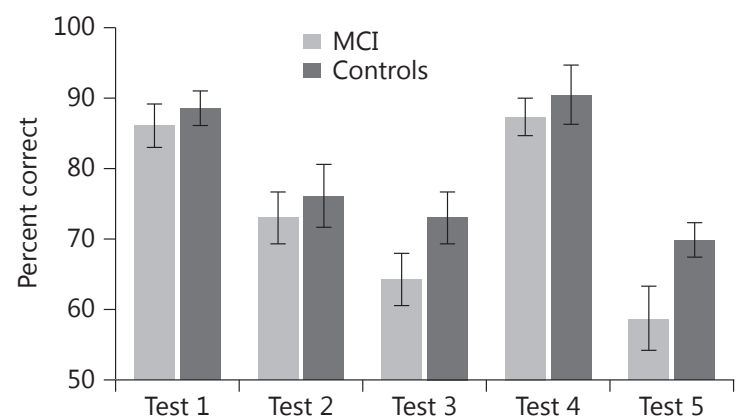

test 1 , although a significant effect of memory load on task performance was found for both participant groups (Table 2; MCI participants, $p=0.04$; controls, $p=0.01$ ), a significant difference in performance between the participant groups was not found for either memory loads (Table 1). No significant recency effects were observed for either memory loads (Table 2 ). The results suggest that MCI participants do not differ significantly from control participants when they are required to remember objects or their locations separately (i.e., in unbound form) during a VSTM task.

In test 3 (object-location binding), which required the participants to explicitly remember objects and their locations combined, a significantly lower performance of MCI participants compared to control participants was found using a 2 (participant groups) $\times 2$ (memory loads) mixed ANOVA (Table 1). The difference was significant at both high and low memory loads (independent-samples $t$ test; Table 1). These results, combined with the non-significant differences found between the participant groups in object-only (test 1) and location-only (test 2) memory, suggest that MCI patients are less adept at binding objects to their locations in VSTM when compared to control participants. A significant effect of memory load on task performance was found for both participant groups (Table 2; MCI participants, $p=0.001$; controls, $p=0.007)$. The recency effects were not significant, except for memory load 2 for MCI participants (Table 2). This is surprising, since with sequence length 4 significant recency effects for explicit memory binding tasks would be expected [36]. However, this result may be because, in some trials, the item probed may have been presented at the first or second temporal position in the memory display, and could have been shown in the test display at the location occupied by the items presented at the third or fourth temporal position. Although the participants may not have been able to remember exactly which temporal position the item probed had originally been presented, they could have used the spatial cue that the probed item had not been shown in the memory display at the location occupied by the items that were presented in the third and fourth temporal positions (due to recency effects for object or location rather than for object-location).

In test 4 , the participants performed an object recognition task, wherein the locations used to present stimuli in the preceding memory display were reused in the test display in both yes and no trials (allowing the effect of location priming to be examined). No significant difference in performance was found between the participant groups using a 2 (participant groups) $\times 2$ (memory loads) mixed ANOVA (Table 1). This suggests that the significant differences observed between the participant groups in test 3 were not confounded by priming effects that may have occurred due to locations being repeated between memory and test displays. Although a significant effect of memory load on task performance was found for 
Sapkota et al.: Patients with Mild Cognitive Impairment Show Lower Visual Short-Term Memory Performance in Feature Binding Tasks

both participant groups (Table 2; MCI participants, $p=0.001$; controls, $p=0.004$ ), the difference in performance between the participant groups was not significant for either memory load (independent-samples $t$ test; Table 1). The recency effects were not significant for either participant group for either memory load (Table 2).

In test 5, the participants' memory for the explicit binding of object locations to their auditorily presented names was measured. The MCI participants performed significantly more poorly than the control participants (independent-samples $t$ test; Table 1). Significant recency effects were observed for both participant groups (Table 2; MCI participants, $p=$ 0.04 ; controls, $p=0.01$ ), implicating global recency effects [37].

Each of the 4 temporal positions in sequence length 4 were probed only once. Consequently, the robustness of the recency effects examined within each participant group may be limited. However, it should be noted that the overall aim of this study was to compare average performance (percent correct responses) between the participant groups for each memory load; 8 trials were collected from each participant for each memory load, except for test 5 , where testing was done only with sequence length 4 and which thus comprised 16 trials. The response biases were not found to differ significantly between the participant groups for any of the tests $(p \geq 0.52)$.

We also examined whether the MCI participants were more impaired than the agematched controls in our VSTM binding tests relative to standard ACE-III memory tests. Overall performance scores were compared between the participant groups for object-location binding (test 3), name-location binding (test 5), and the ACE-III memory tests. Normally aging control participants were found to perform significantly better $(p \leq 0.02)$ than MCI participants by $13.59,19.15$, and $10.39 \%$ for object-location binding, name-location binding, and the ACE-III memory test, respectively. A significant positive correlation in task performance was found between ACE-III and the object-location binding task $(r(18)=0.54, p=0.014)$ and the name-location binding task $(r(18)=0.67, p=0.001)$. Furthermore, test performance in objectlocation binding and crossmodal binding was found to correlate positively with scores for each of the ACE-III subdomains (although the correlations were not statistically significant, $p \geq 0.23$ ). The highest correlation values were observed for the ACE-III memory subdomain $(r(18)=0.20$ with object-location binding; $r(18)=0.28$ with name-location binding). Nonsignificant correlations may have occurred because of the limited number of participants in our group; thus, a larger sample size may show a significant trend.

With a sample size of 10 participants in each group (i.e., 20 in total), the VSTM tests used in this study yielded significant overall differences between $\mathrm{MCI}$ and control participants with a high statistical power of $97 \%$ and an effect size ( $\eta^{2}$ partial) of 0.49 .

\section{Discussion}

We examined the performance profile of $\mathrm{MCI}$ and normally aging control participants in a battery of VSTM tasks. Our objective was to examine the utility of such tasks in discriminating the early cognitive changes found in participants at risk of progressing to dementia from cognitive function in healthily aging controls. Two different types of VSTM binding (object-location and name-location binding) were studied in 5 different memory recognition tests using sequentially presented memory items. Test 1 and test 2 examined VSTM for object identity only and location only, respectively. No significant difference in performance was found between the participant groups in either test. However, a significant difference in performance was found between the participant groups for both memory loads (i.e., sequence lengths) where participants were required to remember an object and its location combined (test 3 ). These results suggest that the MCI participants were less adept at retaining an object- 
location bound unit in VSTM when compared to the age-matched control participants for both high and low memory loads. Furthermore, the MCI participants were found to perform more poorly when asked to retain a name-location bound unit compared to control participants (test 5). To our knowledge, no previous studies have investigated crossmodal binding for name-location using a location recognition task for auditorily presented object names.

One might posit that the differences observed between the participant groups in test 3 may have been driven by differences in priming effects arising from the repeated use of the same locations between the memory and test displays. To address this, in test 4, memory stimulus locations were primed in the test display (in both yes and no trials), but the participants were not required to explicitly remember both the object's identity and its location (since the memory task required object recognition only). No significant difference in performance was found between the participant groups, suggesting that the impairment found in MCI participants for object-location binding was not driven by location priming effects. Earlier researchers have reported no difference in priming effects in VSTM between patients with AD and healthy controls during a picture naming task [36], but a significantly lower performance was found for a color-shape binding task among patients with both early-onset familial AD (caused by E280A mutation of the presenilin-1 gene) and late-onset sporadic AD $[19,38]$.

Our results are supported by the suggestion that medial temporal lobe structures, in particular the hippocampi, which are thought to support memory binding [39], are affected early in those with MCI [40-42], since a significant difference in memory binding performance was found between the MCI and the control participants.

Were our MCI participants more impaired compared to the age-matched controls in the VSTM binding tests than in the ACE-III memory tests? To answer this question, overall performance scores were compared between the participant groups for object-location binding (test 3), name-location binding (test 5), and the ACE-III memory tests. The order of severity of impairment was as follows: name-location binding (it is important to note here that only memory load 4 was used in the name-location binding task, test 5) > object-location binding > ACE-III memory test. A significant positive correlation in task performance was found between ACE-III and the object-location binding task $(r(18)=0.54, p=0.014)$ and the namelocation binding task $(r(18)=0.67, p=0.001)$.

One influential model by Mitchell et al. [12] proposes that age-related differences in VSTM occur due to a decline in our ability to access an object's multiple attributes, such as spatial and temporal features. The unified representation of these multiple features in the brain has also been referred to as a "memory source." We propose that when one feature of an object (its image, name, or spatial or temporal location) is available as a test cue, it will facilitate the retrieval of another bound feature from the memory source. Within this framework, when we consider object-location or name-location binding as a memory source, the provision of one of its components (object, name, or location) as a test cue may benefit memory source retrieval. This may occur because the test cue primes the representation of its (bound) twin object feature stored in VSTM (feature binding priming). We hypothesize that $\mathrm{MCI}$ subjects have a reduced ability to prime and bind the different features of the memory source compared to age-matched control participants.

Jiang et al. [43] suggest that objects in a multi-item visual display are represented hierarchically in the brain, such that the processing of a memory target is influenced by how the surrounding (non-target) objects are configurally arranged. They found that the memory for a target object was enhanced when the spatial configuration of non-target objects was maintained between the memory and test displays. Whether MCI subjects differ significantly in performing VSTM tasks when the stimulus configuration is maintained from the memory to the test display versus not maintained (i.e., if the stimuli occupy the same locations from the 
Sapkota et al.: Patients with Mild Cognitive Impairment Show Lower Visual Short-Term Memory Performance in Feature Binding Tasks

memory to the test display or if they are globally shifted whilst their overall configuration is maintained) is a question for future research.

A possible limitation of our study is that ACE-III was conducted in the Nepali language. A comparison of data from a group of participants who carried out the tests in both English and Nepali versions of ACE-III showed no significant difference in performance between these tests. However, we did not establish ACE-III sensitivity and specificity. Another possible limitation of this study is that we did not include corroborative evidence from imaging to correlate performance with hippocampal volume and/or activity; with the limited resources, this was not possible, but it can form the basis for a future study.

To summarize, our data show that MCI participants exhibit greater impairment in VSTM tasks that require object-location and name-location binding, but not in tasks that do not require memory binding (e.g., object recognition and location recognition). MCI participants are known to be at higher risk for progression to dementia of AD type [44, 45]. A longitudinal follow-up of our participant cohort using the same tests, in combination with other commonly used paper-based tests such as the MMSE or ACE-III, will enable us to verify the apparent efficacy of these VSTM binding tests in the early detection of cognitive decline linked to AD.

\section{Acknowledgement}

This study was funded by the Health and Wellbeing Academy, Anglia Ruskin University, and The South Essex Partnership University National Health Service Foundation Trust (SEPT).

\section{Disclosure Statement}

All the authors have read the papers and have agreed to be listed as authors. We declare that the research was conducted in the absence of any commercial or financial relationships that could be construed as a potential conflict of interest.

\section{References}

1 Rao SC, Rainer G, Miller EK: Integration of what and where in the primate prefrontal cortex. Science 1997;276: 821-824.

2 Ungerleider LG, Mishkin M: Two cortical visual systems; in Ingle DJ, Goodale MA, Mansfield RJW (eds): Analysis of Visual Behavior. Cambridge, MIT Press, 1982, pp 549-586.

-3 Goodale MA, Milner AD: Separate visual pathways for perception and action. Trends Neurosci 1992;15:20-25.

-4 Creem SH, Proffitt DR: Defining the cortical visual systems: "what," "where," and "how." Acta Psychol (Amst) 2001;107:43-68.

5 Bear MF, Connors BW, Paradiso MA: Neuroscience: Exploring the Brain. Philadelphia, Lippincott, Williams \& Wilkins, 2006.

6 Felleman DJ, Van Essen DC: Distributed hierarchical processing in the primate cerebral cortex. Cereb Cortex 1991;1:1-47.

-7 Zanon M, Busan P, Monti F, Pizzolato G, Battaglini PP: Cortical connections between dorsal and ventral visual streams in humans: evidence by TMS/EEG co-registration. Brain Topogr 2010;22:307-317.

-8 Wheeler ME, Treisman AM: Binding in short-term visual memory. J Exp Psychol Gen 2002;131:48-64.

-9 Phillips WA: On the distinction between sensory storage and short-term visual memory. Percept Psychophys 1974;16:283-290.

10 Wheeler ME: Integration of faces and names for memory. Paper presented at the Annual Meeting of the Society for Neuroscience 2000, New Orleans, USA.

11 Sutherland RJ, Rudy JW: Configural association theory: the role of the hippocampal formation in learning, memory, and amnesia. Psychobiology 1989;17:129-144.

12 Mitchell KJ, Johnson MK, Raye CL, Mather M, D’Esposito M: Aging and reflective processes of working memory: binding and test load deficits. Psychol Aging 2000;15;527-541. 
13 Murray EA, Richmond BJ: Role of perirhinal cortex in object perception, memory, and associations. Curr Opin Neurobiol 2001;11:188-193.

14 Hampel H, Bürger K, Teipel SJ, Bokde ALW, Zetterberg H, Blennow K: Core candidate neurochemical and imaging biomarkers of Alzheimer's disease. Alzheimers Dement 2008;4:38-48.

15 Petersen RC, Smith GE, Waring SC, Ivnik RJ, Tangalos EG, Kokmen E: Mild cognitive impairment: clinical characterization and outcome. Arch Neurol 1999;56:303-308.

16 Hsieh S, Schubert S, Hoon C, Mioshi E, Hodges JR: Validation of the Addenbrooke's Cognitive Examination III in frontotemporal dementia and Alzheimer's disease. Dement Geriatr Cogn Disord 2013;36:242-250.

-17 Crawford S, Whitnall L, Robertson J, Evans JJ: A systematic review of the accuracy and clinical utility of the Addenbrooke's Cognitive Examination and the Addenbrooke's Cognitive Examination-Revised in the diagnosis of dementia. Int J Geriatr Psychiatry 2012;27:659-669.

18 Folstein MF, Folstein SE, McHugh PR: "Mini-mental state." A practical method for grading the cognitive state of patients for the clinician. J Psychiatr Res 1975;12:189-198.

19 Gauthier S, Reisberg B, Zaudig M, Petersen RC, Ritchie K, Broich K, et al: Mild cognitive impairment. Lancet 2006;367:1262-1270.

20 Parra MA, Abrahams S, Fabi K, Logie R, Luzzi S, Della Sala S: Short-term memory binding deficits in Alzheimer's disease. Brain 2009;132(pt 4):1057-1066.

21 Maki Y, Yoshida H, Yamaguchi H: Computerized visuo-spatial memory test as a supplementary screening test for dementia. Psychogeriatrics 2010;10:77-82.

22 Sivan AB: Benton Visual Retention Test, ed 5. San Antonio, Psychological Corporation, 1992.

-23 Swainson R, Hodges JR, Galton CJ, Semple J, Michael A, Dunn BD, et al: Early detection and differential diagnosis of Alzheimer's disease and depression with neuropsychological tasks. Dement Geriatr Cogn Disord 2001;12: 265-280.

24 Wechsler D: Wechsler Memory Scale, ed 3. San Antonio, Psychological Corporation, 1997.

25 Rosen WG, Mohs RC, Davis KL: A new rating scale for Alzheimer's disease. Am J Psychiatry 1984;141:13561364.

26 Hughes CP, Berg L, Danziger WL, Coben LA, Martin RL: A new clinical scale for the staging of dementia. Br J Psychiatry 1982;140:566-572.

27 Morris R, Evenden J, Sahakian B, Robbins T: Computer aided assessment of dementia: comparative studies of neuropsychological deficits in Alzheimer type dementia and Parkinson's disease; in Stahl S, Iversen S, Goodman E (eds): Cognitive Neurochemistry. Oxford, Oxford University Press, 1987, pp 21-36.

28 Fowler KS, Saling MM, Conway EL, Semple JM, Louis WJ: Paired associate performance in the early detection of DAT. J Int Neuropsychol Soc 2002;8:58-71.

29 Cullen B, O'Neill B, Evans JJ, Coen RF, Lawlor BA: A review of screening tests for cognitive impairment. J Neurol Neurosurg Psychiatry 2007;78:790-799.

-30 Snodgrass JG, Vanderwart M: A standardized set of 260 pictures: norms for name agreement, image agreement, familiarity, and visual complexity. J Exp Psychol Hum Learn 1980;6:174-215.

-31 Brainard DH: The Psychophysics Toolbox. Spat Vis 1997;10:433-436.

32 Pelli DG: The VideoToolbox software for visual psychophysics: transforming numbers into movies. Spat Vis 1997;10:437-442.

33 Baddeley AD: Working Memory. Oxford, Oxford University Press, 1986

34 Todd JJ, Marois R: Capacity limit of visual short-term memory in human posterior parietal cortex. Nature 2004;428:751-754.

-35 Stanislaw H, Todorov N: Calculation of signal detection theory measures. Behav Res Methods Instrum Comput 1999;31:137-149.

-36 Sapkota RP, van der Linde I, Pardhan S: How does aging affect the types of error made in a visual short-term memory "object-recall” task? Front Aging Neurosci 2015;6:346.

37 Gold JM, Murray RF, Sekuler AB, Bennett PJ, Sekuler R: Visual memory decay is deterministic. Psychol Sci 2005;16:769-774.

-38 Mitchell DB, Schmitt FA: Short- and long-term implicit memory in aging and Alzheimer's disease. Neuropsychol Dev Cogn B Aging Neuropsychol Cogn 2006;13:611-635.

39 Parra MA, Abrahams S, Logie RH, Méndez LG, Lopera F, Della Sala S: Visual short-term memory binding deficits in familial Alzheimer's disease. Brain 2010;133:2702-2713.

40 Hannula DE, Ranganath C: Medial temporal lobe activity predicts successful relational memory binding. J Neurosci 2008;28:116-124.

41 Olson IR, Page K, Moore KS, Chatterjee A, Verfaellie M: Working memory for conjunctions relies on the medial temporal lobe. J Neurosci 2006;26:4596-4601.

-42 Hartley T, Bird CM, Chan D, Cipolotti L, Husain M, Vargha-Khadem F, Burgess N: The hippocampus is required for short-term topographical memory in humans. Hippocampus 2007;17:34-48.

43 Jiang Y, Olson IR, Chun MM: Organization of visual short-term memory. J Exp Psychol Learn Mem Cogn 2002;26:683-702.

44 DeCarli C: Mild cognitive impairment: prevalence, prognosis, aetiology, and treatment. Lancet Neurol 2003;2: $15-21$.

45 Burns A, Zaudig M: Mild cognitive impairment in older people. Lancet 2002;360:1963-1965. 